\title{
Triple-acyclicity in majorities based on difference in support
}

\author{
Bonifacio Llamazares $^{\mathrm{a}, *}$, Patrizia Pérez-Asurmendi ${ }^{\mathrm{b}}$ \\ ${ }^{a}$ PRESAD Research Group, IMUVA, Departamento de Economía Aplicada, Universidad de Valladolid, Avda. Valle de \\ Esgueva 6, 47011 Valladolid, Spain. \\ ${ }^{b}$ PRESAD Research Group, SEED Research Group, IMUVA, Universidad de Valladolid, Avda. Valle de Esgueva 6, 47011 \\ Valladolid, Spain.
}

\begin{abstract}
In this paper we study to what extent majorities based on difference in support leads to triple-acyclic collective decisions. These majorities, which take into account voters' intensities of preference between pairs of alternatives through reciprocal preference relations, require to the winner alternative to exceed the support for the other alternative in a difference fixed before the election. Depending on that difference, i.e., on the threshold of support, and on some requirements on the individual rationality of the voters, we provide necessary and sufficient conditions for avoiding cycles of three alternatives on the collective decision.
\end{abstract}

Keywords: Triple-acyclicity; Majorities based on difference in support; Reciprocal preference relations;

Voting systems

\section{Introduction}

The aggregation of individual preferences under the simple majority rule could lead to a cyclical collective preference, which could prevent the choice of any alternative in the decision process. This fact was firstly pointed out by Condorcet [6] and known since then as the Condorcet's paradox. Recalling the classical example of this paradox, assume the following three voters' preferences over three alternatives $x_{1}, x_{2}$ and $x_{3}:$

$$
x_{1} \succ_{1} x_{2} \succ_{1} x_{3} \quad x_{2} \succ_{2} x_{3} \succ_{2} x_{1} \quad x_{3} \succ_{3} x_{1} \succ_{3} x_{2},
$$

where $x_{i} \succ_{p} x_{j}(i, j, p \in\{1,2,3\})$ means that individual $p$ strictly prefers alternative $x_{i}$ to alternative $x_{j}$ when both alternatives are in comparison. Guided by the preferences given in (1), individuals cast a vote for their preferred alternative in pairwise contests. Aggregating these votes we obtain that alternatives $x_{1}, x_{2}$ and $x_{3}$ defeat $x_{2}, x_{3}$ and $x_{1}$, respectively, by two votes to one. Therefore, the collective preference relation $\succ$ is cyclical (and hence intransitive) given that $x_{1} \succ x_{2} \succ x_{3} \succ x_{1}$. Thus, none of the alternatives is chosen as the collective preferred one.

\footnotetext{
* Corresponding author

Email addresses: boni@eco.uva.es (Bonifacio Llamazares), patrizia.perez@eco.uva.es (Patrizia Pérez-Asurmendi)
} 
In the above example, individual preferences are misrepresented. Every voter declares if he/she prefers an alternative to another one but nothing about the quantification of this preference. A wide variety of authors (see, for instance, Morales [34], Sen [37] or Nurmi [35]) have pointed out the importance for a voting system (for getting a representative aggregation of individual preferences) of taking into account the individuals' intensities of preference among the alternatives in comparison. Reciprocal preference relations formalize such idea. Through them, and by using values in the unit interval, every voter declares his/her intensity of preference between the alternatives compared by pairs. Following this approach, we assume that individuals' preferences are given by reciprocal preference relations and that they fulfill some kind of transitivity condition to avoid misleading preferences.

The introduction of intensities of preference promotes the extension of several aggregation rules to the context of reciprocal preference relations. In the field of majority rules stand out the efforts done by GarcíaLapresta and Llamazares to extend some of them through different operators that aggregate individual reciprocal preferences (see García-Lapresta and Llamazares [16], Llamazares and García-Lapresta [31, 32], and Llamazares $[28,30])$. They have also introduced majorities based on difference in support or $\widetilde{M}_{k}$ majorities (see García-Lapresta and Llamazares [18]). Under them, an alternative $x_{i}$ defeats another one $x_{j}$ if the sum of the voters' intensities of preference for $x_{i}$ exceeds the sum of the intensities of preferences for $x_{j}$ in a given quantity, a threshold $k$, fixed before the election process. These rules extend majorities based on difference of votes (see García-Lapresta and Llamazares [17] and, for the axiomatic characterization, Llamazares [29] and Houy [26]) from the context of ordinary individual preferences to that of intensities of preference.

In the area of ordinary individual preferences, studies about the consistency of several majority rules have been previously done by Greenberg [24], Coughlin [7, 8], Caplin and Nalebuff [2] and Weber [44].

In the area of individual reciprocal preferences, Llamazares et al. [33] have introduced conditions that ensure collective transitivity decisions under majorities based on difference in support. Unfortunately, such conditions require a high support to declare an alternative as a winner regarding highly rational reciprocal preference relations.

In this paper we establish the thresholds $k$ such that majorities based on difference in support do not generate cycles of three alternatives on the collective preference. That is, the conditions that prevent the appearance of the Condorcet's paradox on such rules. Relaxing the collective consistency condition to the weakest one, i.e., triple-acyclicity, promotes the obtaining of more reasonable requirements than in the case of the collective transitivity. To be specific, we will show the following results:

1. The collective preference is triple-acyclic, taking into account 0.5 -transitive reciprocal preference relations, if the threshold is equal to or greater than the integer part of two-thirds the total number of voters. 
2. The collective preference is triple-acyclic, taking into account min-transitive or max-transitive reciprocal preference relations, if the threshold is equal to or greater than one-third the number of voters.

The paper is organized as follows. Section 2 is devoted to introduce the basic technical concepts we deal with. Our main results are stated in sections 3 and 4. Specifically, in Section 3, we set the conditions for triple-acyclic collective decisions under majorities based on difference in support when the rationality of individual preferences is the weakest that it can be, whereas in Section 4 stronger individual rationality conditions than that are taken into account. Finally, Section 5 is dedicated to compare the results obtained here with those on transitive collective decisions under majorities based on difference in support stated in Llamazares et al. [33].

\section{Preliminaries}

This section is organized in three different parts: the first one deals with the types of preference relations concerned, in particular, reciprocal preference relations and ordinary preference relations. The second one is about the consistency conditions asked to these preference relations. Finally, the third one is dedicated to explain the aggregation rule, i.e., majorities based on difference in support.

\subsection{Preference relations}

Consider a set of $m$ voters, $V=\{1, \ldots, m\}$, who show their intensities of preferences on a set of alternatives, $X=\left\{x_{1}, \ldots, x_{n}\right\}$, through reciprocal preference relations $R^{p}: X \times X \longrightarrow[0,1], p=1, \ldots, m$; i.e., $r_{i j}^{p}+r_{j i}^{p}=1$ for all $i, j \in\{1, \ldots, n\}$, where $r_{i j}^{p}=R^{p}\left(x_{i}, x_{j}\right)$. In the context we consider, it is usual to represent $R^{p}$ by an $n \times n$ matrix which coefficients in the unit interval,

$$
R^{p}=\left(\begin{array}{cccc}
r_{11}^{p} & r_{12}^{p} & \ldots & r_{1 n}^{p} \\
r_{21}^{p} & r_{22}^{p} & \ldots & r_{2 n}^{p} \\
\ldots \ldots & \ldots & \ldots & \ldots \\
r_{n 1}^{p} & r_{n 2}^{p} & \ldots & r_{n n}^{p}
\end{array}\right)
$$

By reciprocity, all the main diagonal elements are 0.5 and $r_{j i}^{p}=1-r_{i j}^{p}$ if $j>i$. Therefore,

$$
R^{p}=\left(\begin{array}{cccc}
0.5 & r_{12}^{p} & \ldots & r_{1 n}^{p} \\
1-r_{12}^{p} & 0.5 & \ldots & r_{2 n}^{p} \\
\ldots \ldots \ldots \ldots \ldots & \ldots \ldots \ldots \\
1-r_{1 n}^{p} & 1-r_{2 n}^{p} & \ldots & 0.5
\end{array}\right) .
$$

In this framework, voters can declare their preferences between alternatives, ordered by pairs, but also the degree with which they prefer one alternative to other one through numbers in $[0,1]$. Obviously, they also could declare themselves indifferent between the alternatives. To be more concrete, given two alternatives 
$x_{i}$ and $x_{j}$, if voter $p$ is indifferent between these two alternatives, then $r_{i j}^{p}=0.5$. If he/she absolutely prefers the alternative $x_{i}$ to the alternative $x_{j}$, then $r_{i j}^{p}=1$; on the contrary, if he/she absolutely prefers the alternative $x_{j}$ to $x_{i}$, then $r_{i j}=0$. So far, the preferences described above can be viewed as a representation of ordinary preferences.

Notice that an ordinary preference relation over $X$ is an asymmetric binary relation on $X$ : if $x_{i} P x_{j}$, then does not happen $x_{j} P x_{i}$. The indifference relation associated with $P$ is defined as $x_{i} I x_{j}$ and it means that neither $x_{i}$ is preferred to $x_{j}$ nor $x_{j}$ is preferred to $x_{i}$.

So, every ordinary preference relation $P$ can be considered as a reciprocal preference relation $R$. Furthermore, a reciprocal preference relation $R$ is crisp if $r_{i j}^{p} \in\{0,0.5,1\}$ for all $i, j \in\{1, \ldots, n\}$; so, we can consider ordinary preferences and crisp preferences as equivalent for practical purposes.

Coming back to reciprocal preference relations ${ }^{1}$, they allow voters to describe not so extreme preferences as the absolute preference or the indifference stated above. Specifically, if a voter somewhat prefers alternative $x_{i}$ to $x_{j}$, then $0.5<r_{i j}^{p}<1$ and the closer is this number to 1 , the more $x_{i}$ is preferred to $x_{j}$. On the contrary, if a voter somewhat prefers alternative $x_{j}$ to $x_{i}$, then $0<r_{i j}^{p}<0.5$ and the closer is this number to 0 , the more $x_{j}$ is preferred to $x_{i}$.

Throughout the paper, $\mathcal{R}(X)$ denotes the set of reciprocal preference relations on $X$ and $\mathcal{P}(X)$ denotes the set of ordinary preference relations on $X .^{2}$ Given the set $V$ of $m$ voters, a profile is a vector $\left(R^{1}, \ldots, R^{m}\right)$ which contains the individual preference relations on $X$. The set of profiles is denoted by $\mathcal{R}(X)^{m}$. Moreover, given a set $A, \# A$ will denote the cardinality of $A$. Lastly, given $a \in \mathbb{R},\lfloor a\rfloor$ will denote the integer part of $a$; that is, the highest integer lower than or equal to $a$.

\subsection{Consistency on preference relations}

In the context of ordinary preference relations, it is usual to relate consistency to transitivity condition, but, as said before, in this paper we focus on a weaker consistency property than that, i.e., triple-acyclicity. Triple-acyclicity is obtained by considering the acyclicity condition restricted to three alternatives. This property has been widely studied in the framework of Social Choice theory, specifically in the area of social choice functions (see, among others, Sen [38], Suzumura [39], Schwartz [36] and Cato and Hirata [3]). It provides the minimum consistency requirement for social decisions, that is, to avoid cycles of three alternatives. In the following definitions, we formally recall transitivity, acyclicity and triple-acyclicity conditions.

Definition 1. An ordinary preference relation $P \in \mathcal{P}(X)$ is

\footnotetext{
${ }^{1}$ Note that the property of reciprocity extends the properties of asymmetry (see García-Lapresta and Meneses [19]) and completeness (see De Baets and De Meyer [10]) from the framework of ordinary preferences to the context of intensities of preference.

${ }^{2}$ Unlike in this paper, the notation $\mathcal{P}(X)$ is also used in Ensemble Theory for denoting the set of all subsets of $X$.
} 
1. transitive if for all $x_{i}, x_{j}, x_{l} \in X$ it holds that if $x_{i} P x_{j}$ and $x_{j} P x_{l}$, then it also holds $x_{i} P x_{l}$.

2. acyclic if for all $x_{i_{1}}, \ldots, x_{i_{s}} \in X$ it holds that if $x_{i_{1}} P x_{i_{2}}, \ldots, x_{i_{s-1}} P x_{i_{s}}$, then it does not happen $x_{i_{s}} P x_{i_{1}}$.

3. triple-acyclic if for all $x_{i}, x_{j}, x_{l} \in X$ it holds that if $x_{i} P x_{j}$ and $x_{j} P x_{l}$, then it does not happen $x_{l} P x_{i}$.

Given that transitivity is a stronger condition than acyclicity and that one is a stronger requirement than triple-acyclicity, if a preference relation is not triple-acyclic, it is also not acyclic and, consequently, not transitive. Obviously, acyclicity and triple-acyclicity are equivalent when the social decision involves three alternatives.

In the context of reciprocal preference relations, the notion of transitivity is not as clear as it is in the context of ordinary preference relations (see, for instance, $[4,5,9,10,11,12,15,14,19,20,23,25,27,40$, $41,43,45,46])$.

In our case, we make use of a monotonic operator to define the transitivity conditions for the reciprocal preference relations. Proper definitions are stated below.

Definition 2. A function $g:[0.5,1]^{2} \longrightarrow[0.5,1]$ is a monotonic operator if it satisfies the following conditions:

1. Continuity.

2. Increasingness: $g(a, b) \geq g(c, d)$ for all $a, b, c, d \in[0.5,1]$ such that $a \geq c$ and $b \geq d$.

3. Symmetry: $g(a, b)=g(b, a)$ for all $a, b \in[0.5,1]$.

Definition 3. Given a monotonic operator $g, R \in \mathcal{R}(X)$ is $g$-transitive if for all $i, j, l \in\{1, \ldots, n\}$ the following holds:

$$
\left(r_{i j}>0.5 \text { and } r_{j l}>0.5\right) \Rightarrow\left(r_{i l}>0.5 \text { and } r_{i l} \geq g\left(r_{i j}, r_{j l}\right)\right)
$$

$T_{g}$ denotes the set of all $g$-transitive reciprocal preference relations. Notice that, given two monotonic operators, say $f$ and $g$, such that $f \leq g$, i.e., $f(a, b) \leq g(a, b)$ for all $a, b \in[0.5,1]$, if a reciprocal preference relation $R$ is $g$-transitive, then $R$ is also $f$-transitive; in other words, $T_{g} \subseteq T_{f}$.

We only consider three monotonic operators in order to model the consistency on individual reciprocal preference relations: the constant function 0.5, the minimum and the maximum.

1. $R$ is 0.5 -transitive if $R$ is $g$-transitive, with $g(a, b)=0.5$ for all $a, b \in[0.5,1]$.

2. $R$ is min-transitive if $R$ is $g$-transitive, with $g(a, b)=\min \{a, b\}$ for all $a, b \in[0.5,1]$.

3. $R$ is max-transitive if $R$ is $g$-transitive, with $g(a, b)=\max \{a, b\}$ for all $a, b \in[0.5,1]$.

We denote with $T_{0.5}, T_{\min }, T_{\max }$ the sets of all 0.5 -transitive, min-transitive and max-transitive reciprocal preference relations, respectively. Obviously, $T_{\max } \subseteq T_{\min } \subseteq T_{0.5}$. 


\subsection{Majorities based on difference in support}

Majorities based on difference in support (also called $\widetilde{M}_{k}$ majorities), aggregate individual intensities of preference, i.e., reciprocal preference relations, into collective ordinary preferences. When we compare two alternatives, they declare an alternative as the winner if the sum of the intensities for that alternative exceeds the sum of the intensities for the other one in a threshold, fixed before the election process. Such threshold varies in a continuous space given that intensities also do it. $\widetilde{M}_{k}$ majorities were introduced and axiomatically characterized by García-Lapresta and Llamazares [18] and they generalize majorities based on difference of votes (see García-Lapresta and Llamazares [17] and Llamazares [29]), which ask to the winner alternative to reach a number of votes that exceeds the number of votes for the other alternative in a quantity fixed before the election process. In the following definition we formally present these majorities.

Definition 4. Given a threshold $k \in[0, m)$, the $\widetilde{M}_{k}$ majority is the mapping $\widetilde{M}_{k}: \mathcal{R}(X)^{m} \longrightarrow \mathcal{P}(X)$ defined by $\widetilde{M}_{k}\left(R^{1}, \ldots, R^{m}\right)=P_{k}$, where

$$
x_{i} P_{k} x_{j} \Leftrightarrow \sum_{p=1}^{m} r_{i j}^{p}>\sum_{p=1}^{m} r_{j i}^{p}+k .
$$

As we just show, $\widetilde{M}_{k}$ majorities assign a collective ordinary preference relation to each profile of individual reciprocal preference relations. It is easy to check (see García-Lapresta and Llamazares [18]) that $P_{k}$ can be defined through the average of the individual intensities of preference:

$$
x_{i} P_{k} x_{j} \Leftrightarrow \frac{1}{m} \sum_{p=1}^{m} r_{i j}^{p}>\frac{m+k}{2 m}
$$

or, equivalently,

$$
x_{i} P_{k} x_{j} \Leftrightarrow \sum_{p=1}^{m} r_{i j}^{p}>\frac{m+k}{2} .
$$

The indifference relation associated with $P_{k}$ is defined by:

$$
x_{i} I_{k} x_{j} \Leftrightarrow\left|\sum_{p=1}^{m} r_{i j}^{p}-\sum_{p=1}^{m} r_{j i}^{p}\right| \leq k,
$$

or, equivalently,

$$
x_{i} I_{k} x_{j} \Leftrightarrow\left|\sum_{p=1}^{m} r_{i j}^{p}-\frac{m}{2}\right| \leq \frac{k}{2} .
$$

Some interesting facts could be stated about the behavior of $\widetilde{M}_{k}$ majorities. Assume that an alternative is preferred to another one for a given threshold. If the threshold becomes smaller, then the preference does not change. And if such a threshold becomes greater than before, the preference holds or, at most, turns into indifference. Due to both facts, we have that whenever an alternative is preferred to another one for a certain threshold, such preference cannot be reverse for neither a greater, nor a smaller threshold (see Remark 1 in Llamazares et al. [33]). 


\section{Triple-acyclicity when individuals are 0.5 -transitive}

This section includes the conditions on thresholds $k$ for triple-acyclic collective decisions $P_{k}$ when individual reciprocal relations fulfill 0.5 -transitivity. In such a case, these thresholds depend on the number of voters involved on the election process, which is reflected in the following results.

In Theorem 1 we show that for any threshold smaller than $\lfloor 2 m / 3\rfloor$ we can find profiles of 0.5 -transitive reciprocal preferences for which the triple-acyclicity on collective decision fails.

Theorem 1. There does not exist $k \in[0,\lfloor 2 m / 3\rfloor)$ such that $P_{k}$ is triple-acyclic for every profile of individual preferences $\left(R^{1}, \ldots, R^{m}\right) \in T_{0.5}^{m}$.

Proof. Let $k \in[0,\lfloor 2 m / 3\rfloor)$ and let $R^{\mathrm{I}}, R^{\mathrm{II}}, R^{\mathrm{III}}$ and $R^{\mathrm{IV}}$ be the following reciprocal preference relations:

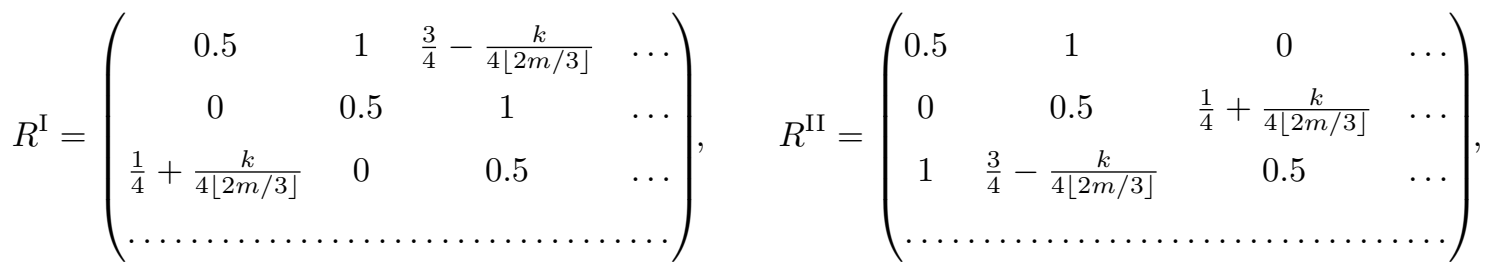

$$
\begin{aligned}
& R^{\mathrm{III}}=\left(\begin{array}{cccc}
0.5 & \frac{1}{4}+\frac{k}{4\lfloor 2 m / 3]} & 0 & \ldots \\
\frac{3}{4}-\frac{k}{4[2 m / 3]} & 0.5 & 1 & \ldots \\
1 & 0 & 0.5 & \ldots \\
\ldots \ldots \ldots \ldots & \ldots \ldots \ldots
\end{array}\right), \quad R^{\mathrm{IV}}=\left(\begin{array}{cccc}
0.5 & 0.5 & 0.5 & \ldots \\
0.5 & 0.5 & 0.5 & \ldots \\
0.5 & 0.5 & 0.5 & \ldots \\
\ldots \ldots & \ldots
\end{array}\right)
\end{aligned}
$$

where non clearly stated elements above take the value of 0.5 . It is easy to check that the previous reciprocal relations belong to $T_{0.5}$. We distinguish three cases:

1. If $m=3 q$, with $q \in \mathbb{N}$, we consider the profile of individual preferences $\left(R^{1}, \ldots, R^{m}\right)$, where

$$
R^{i}= \begin{cases}R^{\mathrm{I}} & \text { if } i=1, \ldots, q, \\ R^{\mathrm{II}} & \text { if } i=q+1, \ldots, 2 q, \\ R^{\mathrm{III}} & \text { if } i=2 q+1, \ldots, 3 q .\end{cases}
$$

According to equivalence (2) and given that $\lfloor 2 m / 3\rfloor=2 q, x_{1} P_{k} x_{2}, x_{2} P_{k} x_{3}$ and $x_{3} P_{k} x_{1}$ will happen if

$$
q\left(1+1+\frac{1}{4}+\frac{k}{8 q}\right)>\frac{3 q+k}{2} .
$$

Since

$$
q\left(1+1+\frac{1}{4}+\frac{k}{8 q}\right)>\frac{3 q+k}{2} \Leftrightarrow 18 q+k>12 q+4 k \Leftrightarrow k<2 q,
$$

$2 q=\lfloor 2 m / 3\rfloor$, and $k<\lfloor 2 m / 3\rfloor$ is satisfied by hypothesis, we get $x_{1} P_{k} x_{2}, x_{2} P_{k} x_{3}$ and $x_{3} P_{k} x_{1}$, and, consequently, $P_{k}$ is not triple-acyclic. 
2. If $m=3 q+1$, with $q \in \mathbb{N}$, we consider the profile of individual preferences $\left(R^{1}, \ldots, R^{m}\right)$, where

$$
R^{i}= \begin{cases}R^{\mathrm{I}} & \text { if } i=1, \ldots, q, \\ R^{\mathrm{II}} & \text { if } i=q+1, \ldots, 2 q, \\ R^{\mathrm{III}} & \text { if } i=2 q+1, \ldots, 3 q, \\ R^{\mathrm{IV}} & \text { if } i=3 q+1 .\end{cases}
$$

According to equivalence (2) and given that $\lfloor 2 m / 3\rfloor=2 q, x_{1} P_{k} x_{2}, x_{2} P_{k} x_{3}$ and $x_{3} P_{k} x_{1}$ will happen if

$$
q\left(1+1+\frac{1}{4}+\frac{k}{8 q}\right)+0.5>\frac{3 q+1+k}{2} .
$$

Since

$$
q\left(1+1+\frac{1}{4}+\frac{k}{8 q}\right)+0.5>\frac{3 q+1+k}{2} \Leftrightarrow 18 q+k>12 q+4 k \Leftrightarrow k<2 q,
$$

$2 q=\lfloor 2 m / 3\rfloor$, and $k<\lfloor 2 m / 3\rfloor$ is satisfied by hypothesis, we get $x_{1} P_{k} x_{2}, x_{2} P_{k} x_{3}$ and $x_{3} P_{k} x_{1}$, and, consequently, $P_{k}$ is not triple-acyclic.

3. If $m=3 q+2$, with $q \in\{0\} \cup \mathbb{N}$, we consider the profile of individual preferences $\left(R^{1}, \ldots, R^{m}\right)$, where

$$
R^{i}= \begin{cases}R^{\mathrm{I}} & \text { if } i=1, \ldots, q+1, \\ R^{\mathrm{II}} & \text { if } i=q+2, \ldots, 2 q+2, \\ R^{\mathrm{III}} & \text { if } i=2 q+3, \ldots, 3 q+2 .\end{cases}
$$

According to equivalence (2) and given that $\lfloor 2 m / 3\rfloor=2 q+1, x_{1} P_{k} x_{2}$ will happen if

$$
q\left(1+1+\frac{1}{4}+\frac{k}{8 q+4}\right)+2>\frac{3 q+2+k}{2} .
$$

On the other hand, $x_{2} P_{k} x_{3}$ and $x_{3} P_{k} x_{1}$ will happen if

$$
q\left(1+1+\frac{1}{4}+\frac{k}{8 q+4}\right)+1+\frac{1}{4}+\frac{k}{8 q+4}>\frac{3 q+2+k}{2} .
$$

Since

$$
\begin{aligned}
q\left(\frac{9}{4}+\frac{k}{8 q+4}\right)+\frac{5}{4}+\frac{k}{8 q+4}>\frac{3 q+2+k}{2} & \Leftrightarrow q\left(9+\frac{k}{2 q+1}\right)+5+\frac{k}{2 q+1}>6 q+4+2 k \\
& \Leftrightarrow 3 q+1>k\left(2-\frac{q+1}{2 q+1}\right) \\
& \Leftrightarrow 3 q+1>k \frac{3 q+1}{2 q+1} \Leftrightarrow k<2 q+1,
\end{aligned}
$$

$2 q+1=\lfloor 2 m / 3\rfloor$, and $k<\lfloor 2 m / 3\rfloor$ is satisfied by hypothesis, we have $x_{2} P_{k} x_{3}$ and $x_{3} P_{k} x_{1}$. Moreover, given that

$$
q\left(1+1+\frac{1}{4}+\frac{k}{8 q+4}\right)+2>q\left(1+1+\frac{1}{4}+\frac{k}{8 q+4}\right)+1+\frac{1}{4}+\frac{k}{8 q+4}
$$

we also have $x_{1} P_{k} x_{2}$. Therefore, $P_{k}$ is not triple-acyclic. 
Triple-acyclic collective decisions are guaranteed when the threshold is greater than or equal to $\lfloor 2 \mathrm{~m} / 3\rfloor$. Before establishing this result, we specify in the following lemma the minimum number of 0.5-transitive individuals who have to prefer an alternative to another one to reach a particular collective intensity of preference for the first alternative over the second one.

Lemma 1. Let $\left(R^{1}, \ldots, R^{m}\right) \in T_{0.5}^{m}$. Given $a \in \mathbb{R}$ and $i, j \in\{1, \ldots, n\}$, if $\sum_{p=1}^{m} r_{i j}^{p}>a$, then there are at least $\lfloor 2 a-m\rfloor+1$ individuals for which $r_{i j}^{p}>0.5$.

Proof. The following case provides the minimum number of individuals for which $r_{i j}^{p}>0.5$ :

1. If $r_{i j}^{p}>0.5$, then $r_{i j}^{p}=1$.

2. If $r_{i j}^{p} \leq 0.5$, then $r_{i j}^{p}=0.5$.

Therefore, in this case, if $z$ is the number of individuals for which $r_{i j}^{p}>0.5$, we have

$$
\sum_{p=1}^{m} r_{i j}^{p}>a \Leftrightarrow 1 z+0.5(m-z)>a \Leftrightarrow 0.5 z>a-0.5 m \Leftrightarrow z>2 a-m \Leftrightarrow z \geq\lfloor 2 a-m\rfloor+1 .
$$

Theorem 2. If $k \in[\lfloor 2 m / 3\rfloor, m)$, then $P_{k}$ is triple-acyclic for every profile of individual preferences $\left(R^{1}, \ldots, R^{m}\right) \in T_{0.5}^{m}$.

Proof. We are going to prove that if $P_{k}$ is not triple-acyclic, then $k<\lfloor 2 m / 3\rfloor$. Suppose there exist $\left(R^{1}, \ldots, R^{m}\right) \in T_{0.5}^{m}$ and $i, j, l \in\{1, \ldots, n\}$ such that $x_{i} P_{k} x_{j}, x_{j} P_{k} x_{l}$ and $x_{l} P_{k} x_{i}$. According to equivalence (2) we have

$$
\sum_{p=1}^{m} r_{i j}^{p}>\frac{m+k}{2}, \quad \sum_{p=1}^{m} r_{j l}^{p}>\frac{m+k}{2} \quad \text { and } \quad \sum_{p=1}^{m} r_{l i}^{p}>\frac{m+k}{2} .
$$

Then, by Lemma 1 , we get

$\#\left\{p \in\{1, \ldots, m\} \mid r_{i j}^{p}>0.5\right\}+\#\left\{p \in\{1, \ldots, m\} \mid r_{j l}^{p}>0.5\right\}+\#\left\{p \in\{1, \ldots, m\} \mid r_{l i}^{p}>0.5\right\} \geq 3(\lfloor k\rfloor+1)$.

On the other hand, $R^{p} \in T_{0.5}$ for every $p \in\{1, \ldots, m\}$. Therefore, for every $p \in\{1, \ldots, m\}$, at most two of the values $r_{i j}^{p}, r_{j l}^{p}$ and $r_{l i}^{p}$ are greater than 0.5 . So,

$$
\#\left\{p \in\{1, \ldots, m\} \mid r_{i j}^{p}>0.5\right\}+\#\left\{p \in\{1, \ldots, m\} \mid r_{j l}^{p}>0.5\right\}+\#\left\{p \in\{1, \ldots, m\} \mid r_{l i}^{p}>0.5\right\} \leq 2 m .
$$

Consequently,

$$
3(\lfloor k\rfloor+1) \leq 2 m \Leftrightarrow\lfloor k\rfloor \leq \frac{2 m}{3}-1 \Leftrightarrow k<\left\lfloor\frac{2 m}{3}\right\rfloor
$$




\section{Triple-acyclicity when individuals are $g$-transitive $(g \geq \min )$}

Now, we explore the conditions for triple-acyclic collective decisions under majorities based on difference in support when reciprocal preference relations are $g$-transitive being $g$ a function greater than or equal to the minimum operator.

Next lemma states that whenever an individual is endowed with the just described reciprocal preference relations over three alternatives, say $x_{i}, x_{j}$ and $x_{l}$, then the sum of the intensities $r_{i j}, r_{j l}$ and $r_{l i}$ reaches at maximum the value of 2 .

Lemma 2. Let $g$ be a monotonic operator such that $g \geq \min$. If $R \in T_{g}$, then $r_{i j}+r_{j l}+r_{l i} \leq 2$ for all $i, j, l \in\{1, \ldots, n\}$.

Proof. Assume, by reduction to absurdity, that there exist $i, j, l \in\{1, \ldots, n\}$ such that $r_{i j}+r_{j l}+r_{l i}>2$. From this inequality we get that at least two of the above addends are greater than 0.5. But, since $R$ is $g$-transitive (being $g$ a function greater than or equal to the minimum), only two of the above addends are greater than 0.5. Assume that $r_{i j}, r_{j l}>0.5$. Then $r_{i l} \geq \min \left\{r_{i j}, r_{j l}\right\}$ or, in the same way, $r_{l i} \leq$ $\max \left\{r_{j i}, r_{l j}\right\}=\max \left\{1-r_{i j}, 1-r_{j l}\right\}$. Therefore,

$$
r_{i j}+r_{j l}+r_{l i} \leq r_{i j}+r_{j l}+\max \left\{1-r_{i j}, 1-r_{j l}\right\} \leq 2,
$$

which contradicts $r_{i j}+r_{j l}+r_{l i}>2$.

With the other two possible cases, say $r_{i j}, r_{l i}>0.5$ and $r_{j l}, r_{l i}>0.5$, the contradiction is also achieved with a similar reasoning as the one just used for the case $r_{i j}, r_{j l}>0.5$.

Now, we can establish a general result for the individual preferences that fulfill the types of transitivities included in this section.

Theorem 3. For each monotonic operator $g$ such that $g \geq \min$ and each $k \in[m / 3, m), P_{k}$ is triple-acyclic for every profile of individual preferences $\left(R^{1}, \ldots, R^{m}\right) \in T_{g}^{m}$.

Proof. Assume, by reduction to absurdity, that $P_{k}$ is not triple-acyclic. Then, it exists a profile of preferences $\left(R^{1}, \ldots, R^{m}\right) \in T_{g}^{m}$ and $i, j, l \in\{1, \ldots, n\}$ such that $x_{i} P_{k} x_{j}, x_{j} P_{k} x_{l}$ and $x_{l} P_{k} x_{i}$; that is,

$$
\sum_{p=1}^{m} r_{i j}^{p}>\frac{m+k}{2}, \quad \sum_{p=1}^{m} r_{j l}^{p}>\frac{m+k}{2} \quad \text { and } \quad \sum_{p=1}^{m} r_{l i}^{p}>\frac{m+k}{2} .
$$

Adding member to member the three inequalities above and taking into account that $k \geq m / 3$, we have

$$
\sum_{p=1}^{m} r_{i j}^{p}+\sum_{p=1}^{m} r_{j l}^{p}+\sum_{p=1}^{m} r_{l i}^{p}>\frac{3}{2}(m+k) \geq \frac{3}{2}\left(m+\frac{m}{3}\right)=2 m
$$

But, by Lemma 2, we have

$$
\sum_{p=1}^{m} r_{i j}^{p}+\sum_{p=1}^{m} r_{j l}^{p}+\sum_{p=1}^{m} r_{l i}^{p}=\sum_{p=1}^{m}\left(r_{i j}^{p}+r_{j l}^{p}+r_{l i}^{p}\right) \leq 2 m
$$


which contradicts inequality (3).

The previous theorem allows us to guarantee triple-acyclic collective decisions when the threshold is greater than or equal to $m / 3$. In what follows, the remaining values of the threshold are analyzed according to whether the reciprocal preference relations fulfill min-transitivity or max-transitivity.

\subsection{The case $g=\min$}

As we establish in the following theorem, when the threshold is smaller than $m / 3$, we can find profiles of min-transitive reciprocal preferences for which the triple-acyclicity on collective decision fails.

Theorem 4. There does not exist $k \in[0, m / 3)$ such that $P_{k}$ is triple-acyclic for every profile of individual preferences $\left(R^{1}, \ldots, R^{m}\right) \in T_{\min }^{m}$.

Proof. Let $k \in[0, m / 3)$ and let $R^{\mathrm{I}}, R^{\mathrm{II}}, R^{\mathrm{II}}, R^{\mathrm{IV}}$ and $R^{\mathrm{V}}$ be the following reciprocal preference relations:

$$
\begin{aligned}
& R^{\mathrm{I}}=\left(\begin{array}{cccc}
0.5 & 1 & \frac{2}{3} & \ldots \\
0 & 0.5 & \frac{2}{3} & \ldots \\
\frac{1}{3} & \frac{1}{3} & 0.5 & \ldots \\
\ldots & \ldots & \ldots & \ldots
\end{array}\right), \quad R^{\mathrm{II}}=\left(\begin{array}{cccc}
0.5 & \frac{1}{3} & 0 & \ldots \\
\frac{2}{3} & 0.5 & \frac{2}{3} & \ldots \\
1 & \frac{1}{3} & 0.5 & \ldots \\
\ldots \ldots & \ldots \ldots \ldots
\end{array}\right), \\
& R^{\mathrm{III}}=\left(\begin{array}{cccc}
0.5 & 1 & 1 & \ldots \\
0 & 0.5 & 1 & \ldots \\
0 & 0 & 0.5 & \ldots \\
\ldots & \ldots & \ldots & \ldots
\end{array}\right), \quad R^{\mathrm{IV}}=\left(\begin{array}{cccc}
0.5 & 1 & 0 & \ldots \\
0 & 0.5 & 0 & \ldots \\
1 & 1 & 0.5 & \ldots \\
\ldots & \ldots & \ldots & \ldots
\end{array}\right), \quad R^{\mathrm{V}}=\left(\begin{array}{cccc}
0.5 & 0 & 0 & \ldots \\
1 & 0.5 & 1 & \ldots \\
1 & 0 & 0.5 & \ldots \\
\ldots & \ldots & \ldots & \ldots
\end{array}\right),
\end{aligned}
$$

where non clearly stated elements above take the value of 0.5 . It is easy to check that the previous reciprocal relations belong to $T_{\min }$. We distinguish two cases according to whether $m$ is even or odd.

1. If $m=2 q$, with $q \in \mathbb{N}$, we consider the profile of individual preferences $\left(R^{1}, \ldots, R^{m}\right)$, where

$$
R^{i}= \begin{cases}R^{\mathrm{I}} & \text { if } i=1, \ldots, q, \\ R^{\mathrm{II}} & \text { if } i=q+1, \ldots, 2 q .\end{cases}
$$

Suppose $(i, j) \in\{(1,2),(2,3),(3,1)\}$. Note that $r_{i j}^{p}+r_{i j}^{q+p}=4 / 3$ for all $p \in\{1, \ldots, q\}$. Therefore,

$$
\sum_{p=1}^{m} r_{i j}^{p}=\sum_{p=1}^{q}\left(r_{i j}^{p}+r_{i j}^{q+p}\right)=\frac{m}{2} \frac{4}{3}=\frac{2 m}{3} .
$$

Now, according to equivalence (2), $x_{1} P_{k} x_{2}, x_{2} P_{k} x_{3}$ and $x_{3} P_{k} x_{1}$ will happen if

$$
\frac{2 m}{3}>\frac{m+k}{2} \Leftrightarrow k<\frac{m}{3},
$$

which is satisfied by hypothesis. Consequently, $P_{k}$ is not triple-acyclic. 
2. If $m=2 q+3$, with $q \in\{0\} \cup \mathbb{N}$, we consider the profile of individual preferences $\left(R^{1}, \ldots, R^{m}\right)$, where

$$
R^{i}= \begin{cases}R^{\mathrm{I}} & \text { if } i=1, \ldots, q, \\ R^{\mathrm{II}} & \text { if } i=q+1, \ldots, 2 q, \\ R^{\mathrm{II}} & \text { if } i=2 q+1, \\ R^{\mathrm{IV}} & \text { if } i=2 q+2, \\ R^{\mathrm{V}} & \text { if } i=2 q+3 .\end{cases}
$$

Suppose $(i, j) \in\{(1,2),(2,3),(3,1)\}$. Note that $r_{i j}^{p}+r_{i j}^{q+p}=4 / 3$ for all $p \in\{1, \ldots, q\}$ and $r_{i j}^{2 q+1}+$ $r_{i j}^{2 q+2}+r_{i j}^{2 q+3}=2$. Therefore,

$$
\sum_{p=1}^{m} r_{i j}^{p}=\sum_{p=1}^{q}\left(r_{i j}^{p}+r_{i j}^{q+p}\right)+2=\frac{m-3}{2} \frac{4}{3}+2=\frac{2 m}{3} .
$$

Now, according to equivalence (2), $x_{1} P_{k} x_{2}, x_{2} P_{k} x_{3}$ and $x_{3} P_{k} x_{1}$ will happen if

$$
\frac{2 m}{3}>\frac{m+k}{2} \Leftrightarrow k<\frac{m}{3},
$$

which is satisfied by hypothesis. Consequently, $P_{k}$ is not triple-acyclic.

\subsection{The case $g=\max$}

Analogously to the case $g=\min$, our aim is to analyze what happens when the threshold is smaller than $m / 3$. But, in this case, we can only show that there exist profiles of max-transitive reciprocal preferences for which the triple-acyclicity on collective decision fails when the threshold is smaller than $\lfloor 2 m / 3\rfloor / 2$.

Theorem 5. There does not exist $k \in[0,\lfloor 2 m / 3\rfloor / 2)$ such that $P_{k}$ is triple-acyclic for every profile of individual preferences $\left(R^{1}, \ldots, R^{m}\right) \in T_{\max }^{m}$.

Proof. Let $k \in[0,\lfloor 2 m / 3\rfloor / 2)$ and let $R^{\mathrm{I}}, R^{\mathrm{II}}, R^{\mathrm{III}}, R^{\mathrm{IV}}, R^{\mathrm{V}}$ and $R^{\mathrm{VI}}$ be the following reciprocal preference relations:

$$
\begin{aligned}
& R^{\mathrm{I}}=\left(\begin{array}{cccc}
0.5 & 1 & 1 & \ldots \\
0 & 0.5 & 1 & \ldots \\
0 & 0 & 0.5 & \ldots \\
\ldots & \ldots & \ldots & \ldots
\end{array}\right), \quad R^{\mathrm{II}}=\left(\begin{array}{cccc}
0.5 & 1 & 0 & \ldots \\
0 & 0.5 & 0 & \ldots \\
1 & 1 & 0.5 & \ldots \\
\ldots \ldots & \ldots & \ldots
\end{array}\right), \quad R^{\mathrm{III}}=\left(\begin{array}{cccc}
0.5 & 0 & 0 & \ldots \\
1 & 0.5 & 1 & \ldots \\
1 & 0 & 0.5 & \ldots \\
\ldots \ldots & \ldots \ldots \ldots
\end{array}\right), \\
& R^{\mathrm{IV}}=\left(\begin{array}{cccc}
0.5 & 0.5 & 0.5 & \cdots \\
0.5 & 0.5 & 0.5 & \ldots \\
0.5 & 0.5 & 0.5 & \ldots \\
\ldots & \ldots & \ldots & \ldots
\end{array}\right), \quad R^{\mathrm{V}}=\left(\begin{array}{cccc}
0.5 & 0.75 & 0.75 & \cdots \\
0.25 & 0.5 & 0.75 & \ldots \\
0.25 & 0.25 & 0.5 & \ldots \\
\ldots \ldots & \ldots & \ldots & \ldots
\end{array}\right), \quad R^{\mathrm{VI}}=\left(\begin{array}{cccc}
0.5 & 0.5 & 0 & \ldots \\
0.5 & 0.5 & 0.5 & \ldots \\
1 & 0.5 & 0.5 & \ldots \\
\ldots \ldots & \ldots & \ldots & \ldots
\end{array}\right),
\end{aligned}
$$


where non clearly stated elements above take the value of 0.5 . It is easy to check that the previous reciprocal relations belong to $T_{\max }$. We distinguish three cases:

1. If $m=3 q$, with $q \in \mathbb{N}$, we consider the profile of individual preferences $\left(R^{1}, \ldots, R^{m}\right)$, where

$$
R^{i}= \begin{cases}R^{\mathrm{I}} & \text { if } i=1, \ldots, q, \\ R^{\mathrm{II}} & \text { if } i=q+1, \ldots, 2 q, \\ R^{\mathrm{III}} & \text { if } i=2 q+1, \ldots, 3 q .\end{cases}
$$

According to equivalence (2), $x_{1} P_{k} x_{2}, x_{2} P_{k} x_{3}$ and $x_{3} P_{k} x_{1}$ will happen if

$$
2 q>\frac{3 q+k}{2} \text {. }
$$

Since

$$
2 q>\frac{3 q+k}{2} \Leftrightarrow k<q,
$$

$q=\lfloor 2 m / 3\rfloor / 2$, and $k<\lfloor 2 m / 3\rfloor / 2$ is satisfied by hypothesis, we get $x_{1} P_{k} x_{2}, x_{2} P_{k} x_{3}$ and $x_{3} P_{k} x_{1}$, and, consequently, $P_{k}$ is not triple-acyclic.

2. If $m=3 q+1$, with $q \in \mathbb{N}$, we consider the profile of individual preferences $\left(R^{1}, \ldots, R^{m}\right)$, where

$$
R^{i}= \begin{cases}R^{\mathrm{I}} & \text { if } i=1, \ldots, q, \\ R^{\mathrm{II}} & \text { if } i=q+1, \ldots, 2 q, \\ R^{\mathrm{III}} & \text { if } i=2 q+1, \ldots, 3 q, \\ R^{\mathrm{IV}} & \text { if } i=3 q+1 .\end{cases}
$$

According to equivalence (2), $x_{1} P_{k} x_{2}, x_{2} P_{k} x_{3}$ and $x_{3} P_{k} x_{1}$ will happen if

$$
2 q+0.5>\frac{3 q+1+k}{2} .
$$

Since

$$
2 q+0.5>\frac{3 q+1+k}{2} \Leftrightarrow k<q,
$$

$q=\lfloor 2 m / 3\rfloor / 2$, and $k<\lfloor 2 m / 3\rfloor / 2$ is satisfied by hypothesis, we get $x_{1} P_{k} x_{2}, x_{2} P_{k} x_{3}$ and $x_{3} P_{k} x_{1}$, and, consequently, $P_{k}$ is not triple-acyclic.

3. If $m=3 q+2$, with $q \in\{0\} \cup \mathbb{N}$, we consider the profile of individual preferences $\left(R^{1}, \ldots, R^{m}\right)$, where

$$
R^{i}= \begin{cases}R^{\mathrm{I}} & \text { if } i=1, \ldots, q, \\ R^{\mathrm{II}} & \text { if } i=q+1, \ldots, 2 q, \\ R^{\mathrm{III}} & \text { if } i=2 q+1, \ldots, 3 q, \\ R^{\mathrm{V}} & \text { if } i=3 q+1, \\ R^{\mathrm{VI}} & \text { if } i=3 q+2 .\end{cases}
$$


According to equivalence (2), $x_{1} P_{k} x_{2}, x_{2} P_{k} x_{3}$ and $x_{3} P_{k} x_{1}$ will happen if

$$
2 q+1.25>\frac{3 q+2+k}{2} .
$$

Since

$$
2 q+1.25>\frac{3 q+2+k}{2} \Leftrightarrow k<q+0.5,
$$

$q+0.5=\lfloor 2 m / 3\rfloor / 2$, and $k<\lfloor 2 m / 3\rfloor / 2$ is satisfied by hypothesis, we get $x_{1} P_{k} x_{2}, x_{2} P_{k} x_{3}$ and $x_{3} P_{k} x_{1}$, and, consequently, $P_{k}$ is not triple-acyclic.

The results obtained in Theorems 3 and 5 do not include all possible values for the threshold $k$. So, we do not know what happens when $k \in[\lfloor 2 m / 3\rfloor / 2, m / 3)$. In the case $m=3 q$, with $q \in \mathbb{N}$, such interval is empty whereas in the cases $m=3 q+1$, with $q \in \mathbb{N}$, and $m=3 q+2$, with $q \in\{0\} \cup \mathbb{N}$, it is not. Specifically, the problematic interval in the case $m=3 q+1$ is $[m / 3-1 / 3, m / 3)$, and $[m / 3-1 / 6, m / 3)$ when $m=3 q+2$. Our conjecture in these intervals is that $P_{k}$ is also triple-acyclic for every profile of max-transitive individual preferences. Below, we show that it is the case when $m=2$, that is, when $m=3 q+2$ with $q=0$. Nevertheless, the mathematical complexity of the proof seems to predict the impossibility of getting similar proofs for the general case $m=3 q+2$ (the same comment can be made for the case $m=3 q+1$ ).

Theorem 6. If $m=2$ and $k \in[0.5,2)$, then $P_{k}$ is triple-acyclic for every profile of individual preferences $\left(R^{1}, R^{2}\right) \in T_{\max }^{2}$.

Proof. Assume, by reduction to absurdity, that $P_{k}$ is not triple-acyclic. Then, there exist $\left(R^{1}, R^{2}\right) \in T_{\max }^{2}$, $i, j, l \in\{1, \ldots, n\}$ such that $x_{i} P_{k} x_{j}, x_{j} P_{k} x_{l}$ and $x_{l} P_{k} x_{i}$. Therefore,

$$
r_{i j}^{1}+r_{i j}^{2}>1+\frac{k}{2} \geq 1.25, \quad r_{j l}^{1}+r_{j l}^{2}>1+\frac{k}{2} \geq 1.25, \quad r_{l i}^{1}+r_{l i}^{2}>1+\frac{k}{2} \geq 1.25 .
$$

It is valuable to highlight that the last condition is equivalent to $r_{i l}^{1}+r_{i l}^{2}<0.75$. Let distinguish three cases depending on the cardinality of the following set:

$$
\mathcal{P}=\left\{p \in\{1,2\} \mid \min \left\{r_{i j}^{p}, r_{j l}^{p}\right\}>0.5\right\}
$$

1. If $\# \mathcal{P}=2$, then, by the max-transitivity condition, we have $r_{i l}^{p} \geq \max \left\{r_{i j}^{p}, r_{j l}^{p}\right\}$ for all $p \in\{1,2\}$. Therefore, $r_{i l}^{1}+r_{i l}^{2} \geq r_{i j}^{1}+r_{i j}^{2}>1.25$, which contradicts $r_{i l}^{1}+r_{i l}^{2}<0.75$.

2. If $\# \mathcal{P}=1$, we can assume, without loss of generality, that $\mathcal{P}=\{1\}$. So, by the max-transitivity condition, we get $r_{i l}^{1} \geq \max \left\{r_{i j}^{1}, r_{j l}^{1}\right\}>0.5$. Given that $r_{i l}^{1}+r_{i l}^{2}<0.75$, we have $r_{i l}^{2}<0.25$ and $\max \left\{r_{i j}^{1}, r_{j l}^{1}\right\}<0.75-r_{i l}^{2}$. We distinguish two cases:

(a) If $r_{i j}^{2} \leq 0.5$, then, $0.75-r_{i l}^{2}>r_{i j}^{1}>1.25-r_{i j}^{2}$; so, $r_{i j}^{2}-r_{i l}^{2}>0.5$, which is an absurdity given that $r_{i j}^{2}, r_{i l}^{2} \in[0,0.5]$. 
(b) If $r_{i j}^{2}>0.5$, then, given that $\mathcal{P}=\{1\}$, we have that $r_{j l}^{2} \leq 0.5$. In that case, $0.75-r_{i l}^{2}>r_{j l}^{1}>$ $1.25-r_{j l}^{2} ;$ so, $r_{j l}^{2}-r_{i l}^{2}>0.5$, which is an absurdity given that $r_{j l}^{2}, r_{i l}^{2} \in[0,0.5]$.

3. If $\# \mathcal{P}=0$, given that $r_{i j}^{1}+r_{i j}^{2}>1.25$ and $r_{j l}^{1}+r_{j l}^{2}>1.25$, we can assume, without lost of generality, that $r_{i j}^{1}>0.5, r_{j l}^{1} \leq 0.5, r_{i j}^{2} \leq 0.5$ and $r_{j l}^{2}>0.5$. Given that $r_{l i}^{1}+r_{l i}^{2}>1.25$, we distinguish three cases:

(a) If $r_{l i}^{1}>0.5$ and $r_{l i}^{2}>0.5$, then, as $R^{1}, R^{2} \in T_{\max }$, we have $r_{l j}^{1} \geq \max \left\{r_{l i}^{1}, r_{i j}^{1}\right\}$ and $r_{j i}^{2} \geq$ $\max \left\{r_{j l}^{2}, r_{l i}^{2}\right\}$. The first condition is equivalent to $r_{j l}^{1} \leq \min \left\{r_{j i}^{1}, r_{i l}^{1}\right\}$. Therefore,

$$
r_{j i}^{1}+r_{j i}^{2} \geq r_{j l}^{1}+r_{j l}^{2}>1.25
$$

which is an absurdity because $r_{i j}^{1}+r_{i j}^{2}>1.25$.

(b) If $r_{l i}^{1}>0.5$ and $r_{l i}^{2} \leq 0.5$, then, as $R^{1} \in T_{\max }$, we have $r_{l j}^{1} \geq \max \left\{r_{l i}^{1}, r_{i j}^{1}\right\}$, which is equivalent to $r_{j l}^{1} \leq \min \left\{r_{j i}^{1}, r_{i l}^{1}\right\}$. Therefore,

$$
r_{j l}^{2}-r_{i l}^{2} \geq\left(r_{j l}^{1}+r_{j l}^{2}\right)-\left(r_{i l}^{1}+r_{i l}^{2}\right)>1.25-0.75=0.5,
$$

which is an absurdity because $r_{j l}^{2}, r_{i l}^{2} \in[0.5,1]$.

(c) If $r_{l i}^{1} \leq 0.5$, then, given that $r_{l i}^{1}+r_{l i}^{2}>1.25$, we have $r_{l i}^{2}>0.75$. As $R^{2} \in T_{\max }$, then $r_{j i}^{2} \geq$ $\max \left\{r_{j l}^{2}, r_{l i}^{2}\right\}$, which is equivalent to $r_{i j}^{2} \leq \min \left\{r_{i l}^{2}, r_{l j}^{2}\right\}$. Therefore,

$$
r_{i j}^{1}-r_{i l}^{1} \geq\left(r_{i j}^{1}+r_{i j}^{2}\right)-\left(r_{i l}^{1}+r_{i l}^{2}\right)>1.25-0.75=0.5,
$$

which is an absurdity because $r_{i j}^{1}, r_{i l}^{1} \in[0.5,1]$.

\section{Discussion}

In this paper we have determined the values of the threshold $k$ to ensure triple-acyclic collective preference relations when we consider $\widetilde{M}_{k}$ majorities on three types of $g$-transitive reciprocal preference relations. On the one hand, for 0.5-transitive reciprocal preference relations, we have found that needed thresholds are, at minimum, around two-thirds of the voters involved in the election process. On the other hand, for mintransitive and max-transitive reciprocal preferences relations, the needed threshold fails to around one-third of the voters. Therefore, the harder the rationality condition over individual preferences is, the smaller the threshold required for triple-acyclic collective decisions is.

It is worth noting that a study of the consistency of the collective decisions under majorities based on difference in support was carried out by Llamazares et al. [33]. In that work, consistency was understood as transitivity. The main conclusions there were somewhat disappointing. On the one hand, for any $k \in[0, m-1)$ and any monotonic operator $g$, we can find profiles of $g$-transitive reciprocal preferences for which the collective preference decision is not transitive. The same result is obtained when $k \in[m-1, m)$ 
and $g(a, b)<(a+b) / 2$ for all $a, b \in[0.5,1], a \neq b$. On the other hand, for $g$-transitive reciprocal preferences, with $g(a, b) \geq(a+b) / 2$ for all $a, b \in[0.5,1]$, transitive collective preferences can be ensured for thresholds located in $[m-1, m)$. Therefore, it is required almost unanimity in individual preferences for arriving to a transitive collective decision and only when individual preference relations fulfill $g$-transitivity, being $g$ greater than or equal to the arithmetic mean operator.

We summarize these results in Table 1, where we show the individual rationality conditions considered in the analysis of triple-acyclicity; that is, 0.5-transitivity, min-transitivity and max-transitivity.

Table 1: Values of $k$ for collective transitivity and triple-acyclicity.

\begin{tabular}{ccc}
\hline Individual $g$-transitivity & Transitivity & Triple-acyclicity \\
\hline$g=0.5$ & $\varnothing$ & {$[\lfloor 2 m / 3\rfloor, m)$} \\
$g=\min$ & $\varnothing$ & {$[m / 3, m)$} \\
$g=\max$ & {$[m-1, m)$} & {$[m / 3, m)$} \\
\hline
\end{tabular}

Notice that the conditions for consistent collective decisions are setting on the thresholds of support and the requirements on them depend on how rational individuals are; i.e., on the transitivity condition that fulfill the individual preferences. So, the more rational the individuals are, the less the needed support for getting consistent collective decisions is. In other words, the stronger the transitivity condition on individual preferences is, the easier to reach consistent collective decisions is. Moreover, the required thresholds look more feasible in the case of triple-acyclicity than in the case of transitivity, given that no so extreme support is required. That is coherent with the fact that triple-acyclicity is a weaker rationality condition than transitivity. Therefore, the innovation of this contribution relies on the finding of less demanding theoretical results to ensure consistent collective decisions than in the case stated in Llamazares et al. [33]. Furthermore, from a practical point of view, we can provide a minimum support that allows us to avoid the Condorcet's paradox in decision problems.

The aggregation of individual reciprocal preferences under majorities based on difference in support can be understood as the aggregation of such individual preference relations through the arithmetic mean operator. Under such view, the preference $P_{k}$ is reached by means of an $\alpha$-cut ${ }^{3}$; i.e., an alternative is preferred to another one if the arithmetic mean of the intensities of preferences for that alternative over the other one exceeds the value of $\alpha$. To rewrite $P_{k}$ by means of an $\alpha$-cut, let $\bar{R}: X \times X \longrightarrow[0,1]$ the reciprocal preference relation defined by the arithmetic mean of the individual intensities of preference, i.e.,

$$
\bar{R}\left(x_{i}, x_{j}\right)=\frac{1}{m} \sum_{p=1}^{m} r_{i j}^{p}
$$

\footnotetext{
${ }^{3}$ If $R \in \mathcal{R}(X)$ and $\alpha \in[0.5,1)$, the $\alpha$-cut of $R$ is the ordinary preference relation $R_{\alpha}$ defined by $x_{i} R_{\alpha} x_{j} \Leftrightarrow R\left(x_{i}, x_{j}\right)>\alpha$.
} 
Then, $P_{k}=\bar{R}_{\alpha}$, with $\alpha=(m+k) / 2 m$.

Notice that cut relations ( $\alpha$-cuts of valued binary relations) have been used by different authors. For instance, Fodor and Roubens [13] give some relationships between valued binary relations and the cut relations associated with them. Świtalski [40], for his part, analyzes relationships between the transitivity and the acyclicity of crisp relations (cut relations and others) obtained from reciprocal preference relations.

It is worth noting that the arithmetic mean operator has been widely used in the literature for aggregating individual intensities of preference into collective intensities of preference. But, as it has been pointed out by some authors, the choice of an alternative has to be unambiguous. Quoting Barrett et al. [1]: In real life, people often have vague preferences... However, when confronted with an actual choice situation, where an alternative has to be chosen from a given feasible set of alternatives, the decision maker must make an unambiguous choice, even when his preferences are fuzzy; there cannot be any vagueness about the actual act of choice itself.

In this sense, $\alpha$-cuts are a valuable tool for obtaining unambiguous choices from collective intensities of preference. The results given in this paper, together with those given by Llamazares et al. [33], allow us to know the values of $\alpha$ for which the collective decision is transitive or triple-acyclic and therefore to provide concrete $\alpha$-cuts to ensure the consistency of the collective decision when considering the arithmetic mean operator to aggregate individual intensities of preferences. These values, calculated by applying the relation between $\alpha$ and $k$ to the values in Table 1, are shown in Table 2 .

Table 2: Values of $\alpha$ for collective transitivity and triple-acyclicity.

\begin{tabular}{ccc}
\hline Individual $g$-transitivity & Transitivity & Triple-acyclicity \\
\hline & & {$[5 / 6,1)$, when $m=3 q$} \\
$g=0.5$ & $\varnothing$ & {$[5 / 6-1 / 3 m, 1)$, when $m=3 q+1$} \\
& & {$[5 / 6-1 / 6 m, 1)$, when $m=3 q+2$} \\
$g=\min$ & $\varnothing$ & {$[2 / 3,1)$} \\
$g=\max$ & {$[1-1 / 2 m, 1)$} & {$[2 / 3,1)$} \\
\hline
\end{tabular}

Finally, previous results on the consistency under majorities based on difference in support rely on the transitivity requirements on the reciprocal preference relations. The impact on the consistency regarding other rationality conditions on reciprocal preference relations (see for instance, Tanino [43], Świtalski [41], Herrera-Viedma et al. [25], De Baets and De Meyer [10] and De Baets et al. [11]) is an open question. Moreover, it could be a significant disparity between the possibility of having cycles and the empirical occurrence of them (see, in similar contexts, Gehrlein and Fishburn [22], Tangian [42] and Gehrlein [21], among others). For this reason, an empirical analysis of such occurrences will be considered in future 
research.

\section{Acknowledgements}

The authors are grateful to José Luis García-Lapresta and three anonymous referees for valuable suggestions and comments. This work is partially supported by the Spanish Ministry of Economy and Competitiveness (Projects ECO2012-32178 and ECO2012-34202) and the Junta de Castilla y León (Consejería de Educación, Project VA066U13).

\section{References}

[1] C. R. Barrett, P. K. Pattanaik, M. Salles, On choosing rationally when preferences are fuzzy, Fuzzy Sets and Systems 34 (1990) 197-212.

[2] A. Caplin, B. Nalebuff, On 64\%-majority rule, Econometrica 56 (1988) 787-814.

[3] S. Cato, D. Hirata, Collective choice rules and collective rationality: a unified method of characterizations, Social Choice and Welfare 34 (2010) 611-630.

[4] F. Chiclana, E. Herrera-Viedma, S. Alonso, F. Herrera, Cardinal consistency of reciprocal preference relations: A characterization of multiplicative transitivity, IEEE Transactions on Fuzzy Systems 17 (2009) 14-23.

[5] F. Chiclana, E. Herrera-Viedma, S. Alonso, R. A. Marques Pereira, Preferences and consistency issues in group decision making, in: H. Bustince, F. Herrera, J. Montero (Eds.), Fuzzy Sets and Their Extensions: Representation, Aggregation and Models, pp. 219-237, Springer-Verlag, Berlin, 2008.

[6] M. de Condorcet, Essai sur l'Application de l'Analyse à la Probabilité des Décisions Rendues à la Pluralité des Voix, Imprimerie Royale, Paris, 1785.

[7] P. J. Coughlin, Necessary and sufficient conditions for $\delta$-relative majority voting equilibria, Econometrica 49 (1981) 12231224.

[8] P. J. Coughlin, Special majority rules and the existence of voting equilibria, Social Choice and Welfare 3 (1986) 31-35.

[9] M. Dasgupta, R. Deb, Transitivity and fuzzy preferences, Social Choice and Welfare 13 (1996) 305-318.

[10] B. De Baets, H. De Meyer, Transitivity frameworks for reciprocal relations: cycle-transitivity versus FG-transitivity, Fuzzy Sets and Systems 152 (2005) 249-270.

[11] B. De Baets, H. De Meyer, B. De Schuymer, S. Jenei, Cyclic evaluation of transitivity of reciprocal relations, Social Choice and Welfare 26 (2006) 217-238.

[12] D. Dubois, H. Prade, Fuzzy Sets and Systems: Theory and Applications, Mathematics in Science and Engineering, Academic Press, New York, 1980.

[13] J. Fodor, M. Roubens, Fuzzy Preference Modelling and Multicriteria Decision Support, Kluwer Academic Publishers, 1994.

[14] S. Freson, B. De Baets, H. De Meyer, Closing reciprocal relations w.r.t. stochastic transitivity, Fuzzy Sets and Systems 241 (2014) 2-26.

[15] S. Freson, H. De Meyer, B. De Baets, Opening reciprocal relations w.r.t. stochastic transitivity, in: S. Greco, B. BouchonMeunier, G. Coletti, M. Fedrizzi, B. Matarazzo, R. R. Yager (Eds.), Advances in Computational Intelligence, volume 300 of Communications in Computer and Information Science, pp. 306-314, Springer, Berlin, 2012.

[16] J. L. García-Lapresta, B. Llamazares, Aggregation of fuzzy preferences: Some rules of the mean, Social Choice and Welfare 17 (2000) 673-690. 
[17] J. L. García-Lapresta, B. Llamazares, Majority decisions based on difference of votes, Journal of Mathematical Economics 35 (2001) 463-481.

[18] J. L. García-Lapresta, B. Llamazares, Preference intensities and majority decisions based on difference of support between alternatives, Group Decision and Negotiation 19 (2010) 527-542.

[19] J. L. García-Lapresta, L. C. Meneses, Individual-valued preferences and their aggregation: consistency analysis in a real case, Fuzzy Sets and Systems 151 (2005) 269-284.

[20] J. L. García-Lapresta, J. Montero, Consistency in preference modelling, in: B. Bouchon-Meunier, G. Coletti, R. R. Yager (Eds.), Modern Information Processing: From Theory to Applications, pp. 87-97, Elsevier, Amsterdam, 2006.

[21] W. V. Gehrlein, Condorcet's Paradox, Springer, 2006.

[22] W. V. Gehrlein, P. C. Fishburn, Condorcet's paradox and anonymous preference profiles, Public Choice 26 (1976) 1-18.

[23] S. Genç, F. E. Boran, D. Akay, Z. Xu, Interval multiplicative transitivity for consistency, missing values and priority weights of interval fuzzy preference relations, Information Sciences 180 (2010) 4877-4891.

[24] J. Greenberg, Consistent majority rules over compact sets of alternatives, Econometrica 47 (1979) 627-636.

[25] E. Herrera-Viedma, F. Herrera, F. Chiclana, M. Luque, Some issues on consistency of fuzzy preference relations, European Journal of Operational Research 154 (2004) 98-109.

[26] N. Houy, Some further characterizations for the forgotten voting rules, Mathematical Social Sciences 53 (2007) $111-121$.

[27] N. Jain, Transitivity of fuzzy relations and rational choice, Annals of Operations Research 23 (1990) 265-278.

[28] B. Llamazares, Simple and absolute special majorities generated by OWA operators, European Journal of Operational Research 158 (2004) 707-720.

[29] B. Llamazares, The forgotten decision rules: Majority rules based on difference of votes, Mathematical Social Sciences 51 (2006) 311-326.

[30] B. Llamazares, Choosing OWA operator weights in the field of Social Choice, Information Sciences 177 (2007) $4745-4756$.

[31] B. Llamazares, J. L. García-Lapresta, Voting systems generated by quasiarithmetic means and OWA operators, in: J. Fodor, B. De Baets (Eds.), Principles of Fuzzy Preference Modelling and Decision Making, pp. 195-213, Academia Press, Ghent, 2003.

[32] B. Llamazares, J. L. García-Lapresta, Extension of some voting systems to the field of gradual preferences, in: H. Bustince, F. Herrera, J. Montero (Eds.), Fuzzy Sets and Their Extensions: Representation, Aggregation and Models, pp. 297-315, Springer-Verlag, Berlin, 2008.

[33] B. Llamazares, P. Pérez-Asurmendi, J. L. García-Lapresta, Collective transitivity in majorities based on difference in support, Fuzzy Sets and Systems 216 (2013) 3-15.

[34] J. I. Morales, Memoria Matemática sobre el Cálculo de la Opinion en las Elecciones, Imprenta Real, Madrid, 1797.

[35] H. Nurmi, Approaches to collective decision making with fuzzy preference relations, Fuzzy Sets and Systems 6 (1981) 249-259.

[36] T. Schwartz, The Logic of Collective Choice, Columbia University Press, New York, 1986.

[37] A. K. Sen, Collective Choice and Social Welfare, Holden-Day, San Francisco, 1970.

[38] A. K. Sen, Social choice theory: A re-examination, Econometrica 45 (1977) 53-89.

[39] K. Suzumura, Rational Choice, Collective Decisions, and Social Welfare, Cambridge University Press, Cambridge, 1983.

[40] Z. Świtalski, Rationality of fuzzy reciprocal preference relations, Fuzzy Sets and Systems 107 (1999) $187-190$.

[41] Z. Świtalski, General transitivity conditions for fuzzy reciprocal preference matrices, Fuzzy Sets and Systems 137 (2003) $85-100$.

[42] A. S. Tangian, Unlikelihood of Condorcet's paradox in a large society, Social Choice and Welfare 17 (2000) $337-365$.

[43] T. Tanino, Fuzzy preference orderings in group decision making, Fuzzy Sets and Systems 12 (1984) $117-131$.

[44] J. S. Weber, An elementary proof of the conditions for a generalized Condorcet paradox, Public Choice 77 (1993) $415-419$. 
[45] Y. Xu, R. Patnayakuni, H. Wang, The ordinal consistency of a fuzzy preference relation, Information Sciences 224 (2013) 152-164.

[46] L. A. Zadeh, Similarity relations and fuzzy orderings, Information Sciences 3 (1971) 177-200. 